\title{
Preparation, Characterization and Pharmacokinetics in Vivo of Oxymatrine-Phospholipid Complex
}

\author{
Peng-Fei YUE ${ }^{1,2}$, Hai-Long YUAN ${ }^{3, *}$, Ming Yang ${ }^{1}$, Wei-Feng ZHU ${ }^{1}$ \\ 1Jiangxi University of Traditional Chinese Medicine,Nanchang 330004 \\ ${ }^{2}$ Key Lab of Modern Preparation of TCM, Ministry of Education, Nanchang 330004 \\ ${ }^{3} 302$ Hospital of PLA\&PLA Institute of Chinese Materia Medica, Beijing 100039
}

\begin{abstract}
The aim of the present study was to prepare oxymatrinephospholipid complex to enhance oral bioavailability of oxymatrine and to study its physicochemical properties and to compare the pharmacokinetic characteristics and bioavailability after oral administration of oxymatrinephospholipid complex in rats. Using tetrahydrofuran as a reaction medium, oxymatrine and phospholipids were resolved into the medium, after the organic solvent was removed under vacuum condition, oxymatrine-phospholipid complex was formed. The new complex's physicochemical properties including differential scanning calorimetry (DSC), X-ray diffraction (XRD), N-octanol/water Partition Coefficient were tested. The concentrations of oxymatrine after oral administration of oxymatrine-phospholipids complex and oxymatrine at different time in rats were determined by HPCE. The pharmacokinetic parameters were computed by software program $3 p 87$. The data showed that oxymatrine and phospholipids in the oxymatrine-phospholipid complex were combined by noncovalent bond, not forming a new compound and the solubility of oxymatrine -phospholipid complex in n-octanol was effectively enhanced. The better hepatocytes permeability was obtained by the phospholipid complex. We found that mean plasma concentration-time curve of oxymatrine after oral administration of oxymatrine-phospholipid complex and oxymatrine in rats was both in accordance with open two-compartment model with firstorder absorption. Pharmacokinetic parameters of oxymatrine, physical mixture and the complex in rats were $T_{\max } 1.71,1.91$ and $2.17 \mathrm{~h}, C_{\max } 0.164,0.247$ and 0.437 $\mu \mathrm{g} \cdot \mathrm{ml}^{-1}, \mathrm{AUC}_{0-\infty} 2.87,3.23$ and $9.43 \mu \mathrm{g} \cdot \mathrm{h} \cdot \mathrm{ml}^{-1}$, respectively. The bioavailability of oxymatrine in rats was increased remarkably after oral administration of oxymatrine-phospholipid complex comparing to oxymatrine and the physical mixture. This was mainly due to an impressive improvement of the lipophilic property of oxymatrine-phospholipid complex.
\end{abstract}

Keywords: Oxymatrine; Phospholipid complex; Physicochemical properties; Pharmacokinetics

\section{Introduction}

Oxymatrine(OMT) is a kind of alkaloid extracted from a Chinese herb (Sophora alopecuraides L.) (Lai et al., 2003). Basic and clinical researches suggested that oxymatrine had the fol- lowing pharmacological effects such as anti-virus, protecting hepatocytes, anti-hepatic fibrosis, immune regulation, etc (Liu et al., 2003; Dong et al., 2003; Xiang et al., 2002; Yang et al., 2002; Chen et al., 2001; Li et al., 1998). In particular, wide attention was paid to its inhibitory effect on hepatitis B virus (HBV) in recent years. Oxymatrine has been proved to have distinct anti-virus effect in the treatment of chronic hepatitis B (CHB). The slight liposolubility of oxymatrine result in the poor permeation across the intestinal epithelial cells and minor the gastrointestinal (GI) tract absorption in rats (Chen et al., 2002; Yu et al., 2002; Chen et al., 2001; Wang et al., 2000). So the wide clinical application of oxymatrine remains a question because of the poor clinical therapeutic effect after oral administration. There are usually several factors responsible for this, but a particularly widespread problem is poor absorption due to slow in the lumen of the gastro-intestinal tract. There are numerous advantages of phospholipids in addition to solubilizing property while considering them for a carrier system. Phospholipids are an important component of cell membrane, having the actions of keeping cell membrane fluidity and treating hepatic disorder. In this paper, OMT-phospholipid complex (OMT-PLC) was studied in order to improve oral bioavailability of OMT.

The objective of this study is: (1) to improve oral bioavailability of OMT, OMT-phospholipid complex was prepared. It is expected that OMT combined with phospholipids might increase the oral bioavailability; (2) the physicochemical characters of OMT-PLC were evaluated, such as DSC, XRD. The n-octanol/water partition coefficient (P) study of OMT-PLC was performed in order to evaluate the improved solubility properties of OMT-PLC in comparison with OMT material and the physical mixture; (3) After oral administration of three formulations: OMT, the physical mixture and OMT-PLC, the pharmacokinetics and bioavailability of OMT in rats were studied. The formulation OMT-PLC, which exhibits comparable to the commercial products or even higher bioavailability, may be clinical candidate for future clinical study.

*Corresponding author: Hai-Long Yuan, 302 Hospital of PLA\&PLA Institute of Chinese Materia Medica, Beijing, China, Tel: 86-10-66933324; Fax: 86-10-63879915; E-mail: ypfpharm@126.com

Received August 19, 2009; Accepted October 23, 2009; Published October 23, 2009

Citation: Yue PF, Yuan HL, Yang M, Zhu WF (2009) Preparation, Characterization and Pharmacokinetics in Vivo of Oxymatrine-Phospholipid Complex. J Bioequiv Availab 1: 099-102. doi:10.4172/jbb.1000015

Copyright: () 2009 Yue PF, et al. This is an open-access article distributed under the terms of the Creative Commons Attribution License, which permits unrestricted use, distribution, and reproduction in any medium, provided the original author and source are credited. 


\section{Journal of Bioequivalence \& Bioavailability - Open Access}

JBB/Vol.1 September-October 2009

\section{Materials and Methods}

\section{Materials}

Oxymatrine was purchased from Ningxia-bo-er-tai-li Ltd, purity $99.13 \%$, and phospholipid was purchased from Hua-qingmei-hen Ltd., and the phosphatidyl content was approximately $82 \%(\mathrm{w} / \mathrm{w})$. The other chemical reagents were of analytical grade or better.

\section{Preparation of Oxymatrine -phospholipid Complexes}

The required amounts of oxymatrine and phospholipids were placed in a $100 \mathrm{ml}$ round-bottom flask and dissolved in tetrahydrofuran. After tetrahydrofuran was evaporated off under vacuum at $40^{\circ} \mathrm{C}$, the dried residues were gathered and placed in desiccators overnight, then crushed in the mortar and sieved with a 100 mesh. The resultant oxymatrine-phospholipid complex was transferred into a glass bottle, flushed with nitrogen and stored in the room temperature.

\section{Determination of the Content of Oxymatrine in Phospho- lipids Complex}

The content of oxymatrine in phospholipids complex was determined as follows. Approximately $5 \mathrm{mg}$ of phospholipids complex were dissolved in $50 \mathrm{ml}$ of solvent A (acetonitrile: dehydrated alcohol $=80: 20, \mathrm{v} / \mathrm{v}$ ), and a $20 \mu \mathrm{l}$ aliquot of the resulting solution was injected into a HPLC system. The stationary phase, $\mathrm{NH}_{2}$ column $(150 \mathrm{~mm} \times 4.6 \mathrm{~mm}, 5 \mu \mathrm{m})$, was kept at $25^{\circ} \mathrm{C}$. The mobile phase was a mixture of acetonitrile : dehydrated alcohol: $3 \% \mathrm{H}_{3} \mathrm{PO}_{4}(80: 10: 10$, v/v). The flow rate was $1.0 \mathrm{ml} / \mathrm{min}$. Effluent was monitored at $220 \mathrm{~nm}$.

\section{Differential Scanning Calorimetry (DSC)}

The samples sealed in the aluminum crimp cell were heated at the speed of $5 \mu \cdot \mathrm{ml}^{-1}$ from 0 to $300 \mathrm{ml}$ in the atmosphere of nitrogen (Dupont 1090B, Dupont, USA). Peak transition onset temperature was determined by means of an analyzer. The peak transition onset temperatures of phospholipids, pure oxymatrine, the mixture of phospholipids and oxymatrine and the oxymatrine-phospholipids complex were compared.

\section{X-ray Diffractometry (XRD)}

The X-ray diffractogram (D/max-r A, Rigaku Denki, Japan) was scanned with the diffraction angle increasing from $5^{\circ}$ to $50^{\circ}, 2 \theta$ angle, with a step angle of $0.04^{\circ}$ and a count time of $1 \mathrm{~s}$.

\section{N-octanol/water Partition Coefficient $(P)$ of OMT-PLC}

P of OMT determination of OMT material, phospholipids complex or the physical mixture was carried out by adding $0.2 \mathrm{~g}$ of OMT material, phospholipids complex or physical mixture to a series of $10 \mathrm{ml}$ water solutions $(\mathrm{pH} 1.5,2.5,3.5,4.5,5.5,6.5$, 7.5 ) in sealed glass containers at $25^{\circ} \mathrm{C}$, respectively. Each experiment was performed in triplicate. All the sixty-three liquids were agitated for $24 \mathrm{~h}$ and centrifuged to remove excessive residues (15 min, $4000 \mathrm{rpm}$ ), respectively. Each liquid was added $10 \mathrm{ml} \mathrm{n}$-octanol and agitated for $24 \mathrm{~h}$. Then they were centrifuged at $4000 \mathrm{rpm}$ for $15 \mathrm{~min}$, respectively. The water phase and n-octanol phase were separated. The water phase and noctanol phase were filtrated through a $0.45 \mu \mathrm{m}$ membrane, respectively. The $1 \mathrm{ml}$ filtrate was mixed with $9 \mathrm{ml}$ of methanol and a $20 \mu \mathrm{l}$ aliquot of the resulting solution was injected into a HPLC and detected as the previous description, the concentrations of OMT were measured, respectively.

$\mathrm{P}$ of OMT of OMT material, physical mixture and phospholipids complex were calculated as follows:

$$
\mathrm{P}=\mathrm{C}_{\mathrm{O}} / \mathrm{C}_{\mathrm{W}}
$$

where $\mathrm{C}_{\mathrm{o}}$ was the concentration of OMT material, OMT-PLC or the physical mixture in n-octanol; $\mathrm{C}_{\mathrm{w}}$ was the concentration of OMT material, OMT-PLC or its physical mixture in water.

\section{Rat Bioavailability Experiments}

\section{Capillary Electrophoresis}

Capillary electrophoresis was performed on a HPCE instrument (Trisep-2010) equipped with a UV detector set at $205 \mathrm{~nm}$ (Luo et al., 1999). Separation and analysis were carried out on an uncoated fused-silica capillary tube $(50 \mu \mathrm{m}$ I.D., $56 \mathrm{~cm}$ total length and $36 \mathrm{~cm}$ from the injection point to the detector) at $25^{\circ} \mathrm{C}$. Before each run, the capillary tube was washed with 0.1 $\mathrm{M} \mathrm{NaOH}$ for $5 \mathrm{~min}$, bidistilled water for $5 \mathrm{~min}$, and then with the operating buffer tris hydroxymethyl aminomethane (Tris,40mM)-sodium dihydrogen phosphate (10 mM)-4\% avantin buffered at $\mathrm{pH} 7.6$ for $5 \mathrm{~min}$. The operating buffer used was degassed by vacuum filtration through a $0.2 \mu \mathrm{m}$ membrane filter, followed by agitation in an ultrasonic bath. The samples to be analyzed were injected automatically, using the pressure injection mode, in which the sample is pressurized for $3 \mathrm{~s}$. The electrophoresis was performed at $20 \mathrm{kV}$ (about $50 \mathrm{~mA}$ ) using normal polarity.

\section{Plasma Sample Preparation and Validity}

The rats were anaesthetized with aether, and $500 \mu$ l blood was taken from the eyeground veins. The plasma was obtained after centrifugation (15 min, $4000 \mathrm{rpm}$ ) was stored at $-20^{\circ} \mathrm{C}$ until analyzed. When the plasma sample was thawed, $50 \mu \mathrm{l}$ of cimetidine solution (CMD, $1.4 \mathrm{mg} \cdot \mathrm{ml}^{-1}$, internal standard), $100 \mu \mathrm{l}$ of $1 \mathrm{M} \mathrm{Na}_{2} \mathrm{CO}_{3}$ solution and $500 \mu \mathrm{l}$ of borate buffer saline $(\mathrm{pH}$ 8.0) were added, and agitated for $30 \mathrm{~s}$. After $4 \mathrm{ml}$ aether was added to the solution above, this mixture was shaken for $3 \mathrm{~min}$ and then centrifuged (15 min, $4000 \mathrm{rpm}$ ). The organic phase was quantitatively decanted into a clear tapered centrifuging tube and the eluate was evaporated under nitrogen at $37^{\circ} \mathrm{C}$. The residues were resuspended in $100 \mu \mathrm{l}$ of mobile phase and centrifuged (15 min, $4000 \mathrm{rpm})$. Aliquots $(20 \mu \mathrm{l})$ of the supernatant were injected for HPCE analysis.

\section{Pharmacokinetic Studies of Oxymatrine-phospholipids Complex and Oxymatrine in Rats}

Eighteen male rats (body weight 180 220g) divided randomly into three groups were fasted for $12 \mathrm{~h}$, but allowed to take water freely. A sample equivalent to $100 \mathrm{mg} / \mathrm{kg}$ of oxymatrine phospholipids complex suspended in $2 \mathrm{ml}$ of water was orally administered to one group of rats. The solutions of oxymatrine and physical mixture equivalent to $100 \mathrm{mg} / \mathrm{kg}$ of oxymatrine were orally administered to the other group of rats,repectively. Peak concentrations $\left(C_{\max }\right)$ and peak times $\left(T_{\max }\right)$ were derived directly from the experimental points. The other pharmacokinetical parameters were computed by software program $3 \mathrm{p} 87$. 


\section{Journal of Bioequivalence \& Bioavailability - Open Access}

JBB/Vol.1 September-October 2009

\section{Results and Discussion}

\section{Preparation of Oxymatrine -phospholipid Complex}

We prepared oxymatrine -phospholipid complex according to different quantity ratio of phospholipids and drugs, such as 1 , 2, 3 and 4. The results showed that when the ratio was more than 3, the appearance of resultant materials appeared viscous and it was not easy that resultant materials were prepared to other preparations, but when the ratio was lower than 3 , the stability of phospholipid complexes was worse and the complex ratio was poor. For the purpose to get the best quality and use the fewest quantity ratio of phospholipids, at last we prepared oxymatrine-phospholipid complex in term of the quantity ratio 3 . The content of oxymatrine in the phospholipids complex was $24.86 \%(\mathrm{w} / \mathrm{w})$.

\section{Differential Scanning Calorimetry}

Figure 1 showed (included as a supplementary information) the DSC curves of phospholipids, oxymatrine physical mixture and phospholipids complex. DSC of phospholipids complex showed the endothermal peaks of oxymatrine and phospholipid are disappeared and the phase transition temperature is lower than the phase transition temperature of phospholipids, it was considered that oxymatrine and phospholipids should have some interaction, such as the combination of hydrogen bonds or van der Waals force (Venema et al., 1988; Lasonder et al., 1990). After the combination of oxymatrine and the phospholipids molecule polarity parts, the carbon-hydrogen chain in phospholipids could turn freely and enwrap the phospholipids molecule polarity parts, which made the sequence decrease between phospholipids aliphatic hydrocarbon chains, made the second endothermal peak of phospholipids disappear and depressed the phase transition temperature.

\section{X-ray Diffractometry}

Figure 2 shows (included as a supplementary information) the powder X-ray diffraction patterns of oxymatrine, phospholipids, their physical mixture and the complex. The oxymatrine powder diffraction pattern shown in Figure 2(c) displayed partial sharp crystalline peaks, which is the characteristic of a molecule with some crystallinity. In contrast, phospholipids shown in Figure2(b) were amorphous lacking crystalline peaks. Compared with that of the physical mixture, the crystalline peaks had disappeared in the complex shown in Figure 2(a). This suggested that oxymatrine in the phospholipids lipid matrix was either molecularly dispersed or amorphous form. However, as seen in Figure 2(d), some crystalline drug signal was still detectable in the physical mixtures of oxymatrine and phospholipids.

\section{N-octanol/water Partition Coefficient $(P)$ of OMT-PLC}

Table 1 showed the n-octanol/water partition coefficient $(\mathrm{P})$ of OMT, the physical mixture and OMT-PLC at different $\mathrm{pH}$. The data showed that OMT-PLC markedly increased the lipophilicify of OMT, and P of OMT-PLC in n-octanol and water was about 10 multiples more than that of OMT material, these were due to the strong dispersibility or/and amorphous form of the phospholipids complexes and polar group of OMT were masked by phospholipids. However, $\mathrm{P}$ of OMT in physical mixture was about 4 multiples more than that of OMT mate-

\begin{tabular}{|l|l|l|l|l|}
\hline \multirow{4}{*}{ samples } & $\mathrm{pH}$ & $\mathrm{Cw} / \mathrm{g} \cdot \mathrm{ml}^{-1}$ & $\mathrm{Co} / \mathrm{g} \cdot \mathrm{ml}^{-1}$ & $\begin{array}{l}\mathrm{P} \\
(\mathrm{Co} / \mathrm{CW})\end{array}$ \\
\hline \multirow{5}{*}{ OMT } & 1.5 & $0.183 \pm 0.0098$ & $0.022 \pm 0.0038$ & 0.120 \\
\cline { 2 - 5 } & 2.5 & $0.175 \pm 0.0086$ & $0.024 \pm 0.0046$ & 0.137 \\
\cline { 2 - 5 } & 3.5 & $0.173 \pm 0.0034$ & $0.023 \pm 0.0073$ & 0.133 \\
\cline { 2 - 5 } & 4.5 & $0.165 \pm 0.0007$ & $0.034 \pm 0.0057$ & 0.206 \\
\cline { 2 - 5 } & 5.5 & $0.168 \pm 0.0097$ & $0.037 \pm 0.0068$ & 0.220 \\
\cline { 2 - 5 } mixture & 6.5 & $0.164 \pm 0.0086$ & $0.031 \pm 0.0038$ & 0.189 \\
\cline { 2 - 5 } & 7.5 & $0.167 \pm 0.0067$ & $0.033 \pm 0.0043$ & 0.197 \\
\hline \multirow{5}{*}{ OMT-PLC } & 1.5 & $0.136 \pm 0.0076$ & $0.066 \pm 0.0075$ & 0.485 \\
\cline { 2 - 5 } & 2.5 & $0.137 \pm 0.0084$ & $0.069 \pm 0.0037$ & 0.503 \\
\cline { 2 - 5 } & 3.5 & $0.139 \pm 0.0039$ & $0.068 \pm 0.0028$ & 0.489 \\
\cline { 2 - 5 } & 4.5 & $0.133 \pm 0.0059$ & $0.066 \pm 0.0063$ & 0.496 \\
\cline { 2 - 5 } & 5.5 & $0.142 \pm 0.0065$ & $0.057 \pm 0.0052$ & 0.401 \\
\cline { 2 - 5 } & 6.5 & $0.148 \pm 0.0098$ & $0.055 \pm 0.0084$ & 0.372 \\
\cline { 2 - 5 } & 7.5 & $0.147 \pm 0.0056$ & $0.047 \pm 0.0057$ & 0.320 \\
\cline { 2 - 5 } & 1.5 & $0.074 \pm 0.0050$ & $0.125 \pm 0.0063$ & 1.69 \\
\cline { 2 - 5 } & 2.5 & $0.063 \pm 0.0185$ & $0.133 \pm 0.0057$ & 2.11 \\
\cline { 2 - 5 } & 4.5 & $0.064 \pm 0.0044$ & $0.127 \pm 0.0052$ & 1.98 \\
\cline { 2 - 5 } & 6.5 & $0.065 \pm 0.0050$ & $0.138 \pm 0.0057$ & 2.091 \\
\cline { 2 - 5 } & 7.5 & $0.066 \pm 0.0089$ & $0.129 \pm 0.0036$ & 1.984 \\
\hline \multirow{5}{*}{ Tical } & $0.128 \pm 0.0073$ & 2.0 \\
\hline
\end{tabular}

Table 1: N-octanol/water partition coefficient (P) of oxymatrine, physical mixture and OMT-PLC at different $\mathrm{pH}$. Values are mean \pm S.D. $(n=3)$.

rial, because the phospholipids slightly improved the hydrophilicity and lipophilicify of OMT in physical mixture by means of its solubilization.

\section{Rats Bioavailability}

Oxymatrine in plasma was completely separated under analytical conditions (Figure 3) (included as a supplementary information). The calibration curve was found to be linear 13.501$0.046(r=0.9992$, where $x$ is the concentration ratio of OTM to $\mathrm{CA}$ and $\mathrm{y}$ is the corresponding peak-area ratio UDCA/CA) in the 0.0179 to $0.1790 \mathrm{mg} \cdot \mathrm{ml}^{-1}$ range. The results attained from the method recoveries of high, middle and low concentrations were $85.15,87.11$ and $88.37 \%$, respectively. The R.S.D. in days were $3.18,3.52$ and $3.29 \%$, respectively, the R.S.D. intra-days were $3.41,3.74$ and $3.45 \%$, respectively, which showed recoveries and RSD in days or intra-days were satisfying, and the

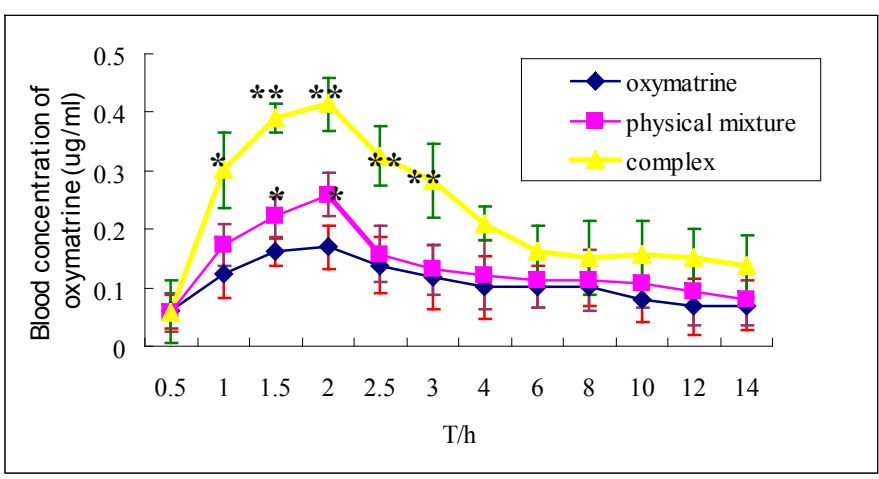

Figure 4: Mean plasma concentration-time curve of oxymatrine in rats after oral administration of oxymatrine-phospholipid complex, oxymatrine and physical mixture equivalent to 100 $\mathrm{mg} / \mathrm{kg}$ of oxymatrine $(\mathrm{n}=6)$, respectively. Values are mean $\pm \mathrm{SD}$ $(\mathrm{n}=6 /$ group/time point $) . * \mathrm{P}<0.05$ and $* * \mathrm{P}<0.01$ are statistical significances with the OMT-PLC versus OMT or physical mixture.
J Bioequiv Availab
Volume 1(3): 099-102 (2009) - 101

ISSN:0975-0851 JBB, an open access journal 


\section{Journal of Bioequivalence \& Bioavailability - Open Access}

JBB/Vol.1 September-October 2009

lowest detection limit was $35 \mathrm{ng} \cdot \mathrm{ml}^{-1}$.

Figure 4 showed the sample equivalent to $100 \mathrm{mg} / \mathrm{kg}$ of oxymatrine of phospholipids complex and oxymatrine was respectively orally administered to rats $(n=6)$. From the above profile and Table 2 , it was known that the average value of $C_{\max }$ is $0.437 \mu \mathrm{g} \cdot \mathrm{ml}^{-1}$ after oral administration of phospholipids complex with a $T_{\max }$ of about $2.17 \mathrm{~h}$. However, the average value of $C_{\max }$ was $0.164 \mu \mathrm{g} \cdot \mathrm{ml}^{-1}$ after oral administration of oxymatrine solution with a $T_{\max }$ of about $1.71 \mathrm{~h}$, the average value of $C_{\max }$ was $0.247 \mu \mathrm{g} \cdot \mathrm{ml}^{-1}$ after oral administration of oxymatrine solution with a $T_{\max }$ of about $1.91 \mathrm{~h}$. The average value of $\mathrm{AUC}_{0-\infty}$ of oxymatrine, the phtsical mixture and the complex in rats were $2.87,3.23$ and $9.43 \mu \mathrm{g} \cdot \mathrm{h} \cdot \mathrm{ml}^{-1}$, respectively. And the bioavailability of oxymatrine was 3.29 multiples than oxymatrine. The pharmacokinetical data were simulated by non-linear least squares. The results showed that open two-compartment model and 1st-order absorption were fitted to both phospholipids complex and oxymatrine plasma concentration-time course in vivo of rats.

\begin{tabular}{|c|c|c|c|}
\hline Parameters & Oxymatrine & Physical mixture & Complex \\
\hline $\begin{array}{c}\mathrm{AUC}_{0-24} \\
\left(\mu \mathrm{g} \cdot \mathrm{h} \cdot \mathrm{ml}^{-1}\right)\end{array}$ & $1.97 \pm 0.218$ & $2.42 \pm 0.371$ & $6.21 \pm 0.859 *$ \\
\hline $\begin{array}{c}\mathrm{AUC} \mathrm{C}_{0-\infty} \\
\left(\mu \mathrm{g} \cdot \mathrm{h} \cdot \mathrm{ml}^{-1}\right)\end{array}$ & $2.87 \pm 0.417$ & $3.23 \pm 0.317$ & $9.43 \pm 0.384 *$ \\
\hline $\begin{array}{c}\mathrm{CL} \\
\left(\mathrm{mLh}^{-1}\right)\end{array}$ & $4.72 \pm 0.45$ & $4.83 \pm 0.76$ & $4.97 \pm \pm 0.85$ \\
\hline $\begin{array}{c}\mathrm{T}_{\max } \\
(\mathrm{h})\end{array}$ & $1.71 \pm 0.54$ & $1.91 \pm 0.64$ & $2.17 \pm 0.46$ \\
\hline $\begin{array}{c}\mathrm{C}_{\max } \\
\left(\mu \mathrm{g} \cdot \mathrm{ml}^{-1}\right)\end{array}$ & $0.164 \pm 0.045$ & $0.247 \pm 0.075$ & $0.437 \pm 0.083 *$ \\
\hline
\end{tabular}

Table 2: The main pharmacokinetic parameters of phospholipids complex ,physical mixture and oxymatrine in rats $(n=6)$. ${ }^{*} \mathrm{P}$ $<0.05$ are statistical significances with the OMT-PLC versus OMT or physical mixture.

\section{Conclusion}

In this study, OMT-PLC was successfully prepared by a simple method. DSC and XRD curves of phospholipids complex showed that OMT and phospholipids combined and formed some kind bond, such as hydrogen bonds or van der Waals force. The $\mathrm{N}$-octanol/water partition coefficient(P) of OMT-PLC studies showed OMT-PLC surprisingly increased the hydrophilicity and lipophilicify of OMT, and P of OMT-PLC in n-octanol and water was about 10 multiples more than that of UCDA material. The blood concentration of OMT was precisely assayed by HPCE Compared with OMT material and the physical mixture, the phospholipid complex can markedly improve the bioavailability of OMT in vivo of rats. It would be further studied about the absorbed mechanism of OMT-PLC through small intestine and therapeutic evaluation in vivo. The OMT-PLC would be more prospective preparation in future.

\section{Acknowledgements}

The authors wish to thank the Chinese Medicine Modernization Foundation of Shanghai City for financial support (No. 05DZ19711).

\section{References}

1. Chen Y, Li J, Zeng M, Lu L, Qu D, et al. (2001) The inhibi- tory effect of oxymatrine on hepatitis $\mathrm{C}$ virus in vitro. Zhonghua Ganzangbing Zazhi 9: 12-14. » Pubmed » Google Scholar

2. Chen XS, Wang GJ, Cai X, Yu HY, Hu YP (2001) Inhibition of hepatitis B virus by oxymatrine in vivo. World J Gastroenterol 7: 49-52.» CrossRef » Pubmed » Google Scholar

3. Chen YX, Mao BY, Jiang JH (2002) Relationship between serum load of HBV-DNA and therapeutic effect of oxymatrine in patients with chronic hepatitis B. Zhongguo Zhongxiyi Jiehe Zazhi 22: 335-336. » Pubmed » Google Scholar

4. Dong Y, Xi H, Yu Y, Wang Q, Jiang K, et al. (2002) Effects of oxymatrine on the serum levels of $\mathrm{T}$ helper cell 1 and 2 cytokines and the expression of the $\mathrm{S}$ gene in hepatitis $\mathrm{B}$ virus $S$ gene transgenic mice: a study on the anti-hepatitis $B$ virus mechanism of oxymatrine. J Gastroenterol Hepatol 17: 1299-1306.» CrossRef » Pubmed » Google Scholar

5. Lai JP, He XW, Jiang Y, Chen F (2003) Preparative separation and determination of matrine from the Chinese medicinal plant Sophora flavescens Ait by molecularly imprinted solid-phase extraction. Anal Bioanal Chem 375: $264-$ 269. » CrossRef » Pubmed » Google Scholar

6. Lasonder E, Weringa WD (1990) An NMR and DSC study of the interaction of phospholipids vesicles with some antiinflammatory agents. J Colloid Interface Sci 139: 469-478. " CrossRef » Google Scholar

7. Li J, Li C, Zeng M (1998) Preliminary study on therapeutic effect of oxymatrine in treating patients with chronic hepatitis C. Zhongguo Zhongxiyi Jiehe Zazhi 18: 227-229. » Pubmed " Google Scholar

8. Liu J, Manheimer E, Tsutani K, Gluud C (2003) Medicinal herbs for hepatitis $\mathrm{C}$ virus infection: a Cochrane hepatobiliary systematic review of randomized trials. Am J Gastroenterol 98: 538-544.» CrossRef » Pubmed » Google Scholar

9. Luo M, He P, Wu M (1999) Determination of Matrine and Oxymatrine by HPCE. J Chinese Traditional and Herbal Drugs 30: 261-263.

10. Venema FR, Weringa WD (1988) The interactions of phospholipid vesicles with some anti-inflammatory agents. J Colloid Interface Sci 125: 484-500. » CrossRef» Google Scholar

11. Wang BE (2000) Treatment of chronic liver diseases with traditional Chinese medicine. J Gastroenterol Hepatol 15: E67-70.» CrossRef » Pubmed » Google Scholar

12. Xiang X, Wang G, Cai X, Li Y (2002) Effect of oxymatrine on murine fulminant hepatitis and hepatocyte apoptosis. Chin Med J 115: 593-596. » CrossRef » Pubmed » Google Scholar

13. Yang W, Zeng M, Fan Z, Mao Y, Song Y, et al. (2002) Prophylactic and therapeutic effect of oxymatrine on D-galactosamine-induced rat liver fibrosis. Zhonghua Ganzangbing Zazhi 10: 193-196. » Pubmed » Google Scholar

14. Yu YY, Wang QH, Zhu LM, Zhang QB, Xu DZ, et al. (2002) A clinical research on oxymatrine for the treatment of chronic hepatitis B. Zhonghua Ganzangbing Zazhi 10: 280-281. » Pubmed » Google Scholar 